\title{
Comportamento do concreto submetido a elevadas temperaturas
}

\author{
Behavior of concrete subjected to high \\ temperatures
}

\author{
Alisson Rodrigues de Oliveira Dias ${ }^{1}$, Felipe Alves Amancio ${ }^{1}$, \\ Maria Fabíola de Carvalho Rafael ${ }^{1}$, Antônio Eduardo Bezerra Cabral ${ }^{2}$
}

\begin{abstract}
${ }^{1}$ Programa de Pós-Graduação em Engenharia Civil - Estruturas e Construção Civil - PEC, Universidade Federal do Ceará, CEP: 60455-900, Fortaleza, CE, Brasil.

e-mail: rodrigues_alisson@live.com, felipeaamancio@hotmail.com, mfabiolacr@gmail.com

${ }^{2}$ Programa de Pós-Graduação em Engenharia Civil - Estruturas e Construção Civil - PEC, Universidade Federal do Ceará, CEP: 60455-900, Fortaleza, CE, Brasil.

e-mail: eduardo.cabral@ufc.br
\end{abstract}

\section{RESUMO}

A resposta ao fogo de elementos estruturais depende das propriedades térmicas, mecânicas e de deformação do concreto. Estas propriedades variam significativamente com a temperatura e dependem da composição e características do concreto. Com isso, o presente trabalho teve como objetivo realizar uma análise do comportamento do concreto submetido a elevadas temperaturas. Para isso, os programas experimentais adotados visaram investigar o comportamento do módulo de elasticidade do concreto, a resistência à compressão residual conforme o tipo de resfriamento, tempo de exposição, $o f_{c k}$ do concreto e o teor de adição de fibra de polipropileno, além da análise da perda de massa e da influência na velocidade do pulso ultrassônico. As temperaturas as quais foram submetidas o concreto foram $200{ }^{\circ} \mathrm{C}, 400{ }^{\circ} \mathrm{C}, 600{ }^{\circ} \mathrm{C}$ e $800^{\circ} \mathrm{C}$. Pode-se concluir que o concreto quando exposto a elevadas temperaturas sofre deterioração significativa, como redução do módulo de elasticidade e da resistência à compressão, spalling do concreto e consequentemente perda de durabilidade. Foi observado que a adição de fibras de polipropileno ajudou a prevenir o spalling em temperaturas até $600{ }^{\circ} \mathrm{C}$. Além disso, com base em testes estatísticos, os resultados demonstraram que o tipo de resfriamento não influencia na resistência residual, já o tempo de exposição, o tipo de $f_{c k}$ e o teor de adição de fibras de polipropileno influenciam significativamente.

Palavras-chave: Concreto; Elevadas temperaturas; Resistência à compressão.

\section{ABSTRACT}

The fire response of structural elements depends on the thermal, mechanical and deformation properties of concrete. The properties vary significantly with temperature and depend on the composition and characteristics of the concrete. Thus, the objective of this work was to perform an analysis of the behavior of concrete subjected to high temperatures. For this, the experimental programs adopted were to investigate the behavior of the modulus of elasticity of the concrete, the resistance to the residual compression according to the type of cooling, exposure time, the concrete $\mathrm{f}_{\mathrm{ck}}$ and the addition content of polypropylene fiber, besides of the analysis of mass loss and ultrasonic pulse velocity. The temperatures at which the concrete was subjected were $200{ }^{\circ} \mathrm{C}, 400{ }^{\circ} \mathrm{C}, 600{ }^{\circ} \mathrm{C}$ and $800{ }^{\circ} \mathrm{C}$. It can be concluded that concrete exposed to high temperatures undergoes significant deterioration such as reduction of modulus of elasticity and compressive strength, spalling of concrete and consequently loss of durability. The addition of polypropylene fibers helped prevent spalling at temperatures up to $600{ }^{\circ} \mathrm{C}$. Furthermore, based on statistical tests, the results showed that the A type of cooling does not influence the residual resistance, since the exposure time, the type of $f_{c k}$ and the addition content of polypropylene fibers influence significantly.

Keywords: Concrete; High temperatures; Compressive strength. 


\section{INTRODUÇÃO}

A engenharia civil enfrenta um imenso desafio em decorrência de danos causados pela ação do fogo durante a fase de construção e de serviço. Nos últimos anos, o surgimento de manifestações patológicas e danos estruturais relacionados com o fogo são freqüentemente relatados em todo o mundo, ameaçando seriamente a segurança pessoal e da propriedade [1]. Os danos causados por um incêndio a uma estrutura de concreto podem ir desde uma simples descoloração ou mancha produzida pela fumaça, à completa destruição do elemento por perda de sua resistência mecânica [2].

Isso ocorre porque o fogo representa uma das condições deletérias mais severas às quais as estruturas possam ser submetidas. Sendo assim, a previsão de medidas adequadas de segurança contra incêndio é um aspecto importante do projeto de construção [3,4].

O concreto quando submetido a elevadas temperaturas, como no caso dos incêndios, sofre significativas mudanças físico-químicas $[4,5,6,7,8]$. Esta exposição pode ocasionar deteriorações relevantes, como a perda de resistência, redução do módulo de elasticidade e degradação da durabilidade no concreto $[9,10,11,12]$ além de fissurações, desprendimento da massa de concreto superficial, destruição da ligação entre a pasta de cimento e os agregados e a deterioração gradual da pasta de cimento endurecida [6]. Essa é principalmente causada pelas diferenças nos coeficientes de expansão térmica dos materiais constituintes, como a pasta de cimento, os agregados etc., bem como está relacionada às mudanças dos materiais constituintes do concreto em temperaturas muito elevadas [13].

As manifestações patológicas ocasionadas pela ação das altas temperaturas são geralmente visíveis sob a forma de rachaduras na superfície e o efeito denominado spalling [14]. Spalling é definido como a quebra de camadas (peças) de concreto a partir da superfície quando exposto a temperaturas elevadas e em rápido aumento, como as encontradas nos incêndios. Este desprendimento pode ocorrer logo após a exposição ao aquecimento rápido e pode ser acompanhado por explosões violentas ou acontecer durante fases posteriores de fogo quando a peça se tornou tão fraca após o aquecimento de modo que, quando as rachaduras se desenvolvem, os pedaços de concreto caem da superfície. As conseqüências são limitadas, desde que a extensão do dano seja pequena, mas um spalling extensivo pode levar à perda precoce de estabilidade e integridade da estrutura [4].

Apesar de o concreto de alta resistência contribuir para uma melhor durabilidade e capacidade de carga de uma estrutura, em comparação ao concreto de resistência normal, são mais sensíveis ao spalling por causa de sua matriz de alta densidade e pequena permeabilidade $[15,16,17]$. Em relação a adição de fibras em matrizes cimentícias, o uso de fibras de polipropileno no concreto reduz e até evita o spalling quando submetido a elevadas temperaturas $[16,17,18,19,20]$, porém esta adição afeta negativamente a trabalhabilidade no estado fresco $[21,22]$.

Quanto aos valores de resistência residual do concreto produzido com fibra de polipropileno e submetido a elevadas temperaturas, há controvérsias. Enquanto estudos demonstram que essa resistência em concretos contendo fibras foram maiores que as de concretos sem essa adição após exposição [23], outros demonstram que a adição teve efeito negativo sobre as propriedades mecânicas residuais do concreto [1, 16, 24, 25].

Neste sentido, o presente trabalho visou analisar o comportamento do concreto submetido a elevadas temperaturas, variando parâmetros como tempo de exposição, tipo de resfriamento, $f_{c k}$ e o teor de fibras de polipropileno.

\section{MATERIAIS E MÉTODOS}

\subsection{Materiais}

Foi utilizado cimento Portland CPII-Z 32. As características do cimento utilizado são apresentadas na Tabela 1. O agregado graúdo era de origem granítica e o agregado miúdo silicoso.

Tabela 1: Características físicas e químicas do cimento utilizado.

\begin{tabular}{l|l|l}
\hline PROPRIEDADES QUÍMICAS & RESULTADO & ESPECIFICAÇÃO - NBR 16697:2018 \\
\hline Perda ao fogo $(\%)$ & 5,79 & $\leq 8,5 \%$ \\
\hline Óxido de Magnésio $-\mathrm{MgO}(\%)$ & 2,77 & - \\
\hline Trióxido de Enxofre $-\mathrm{SO}_{3}(\%)$ & 3,21 & $\leq 4,5 \%$ \\
\hline
\end{tabular}




\begin{tabular}{|c|c|c|c|}
\hline \multicolumn{2}{|l|}{ Resíduo Insolúvel (\%) } & 14,42 & $\leq 18,5 \%$ \\
\hline \multicolumn{2}{|l|}{ PROPRIEDADES FÍSICAS } & RESULTADO & ESPECIFICAÇÃO - NBR 16697:2018 \\
\hline \multicolumn{2}{|l|}{ Área específica (Blaine) $\left(\mathrm{cm}^{2} / \mathrm{g}\right)$} & 5065 & $\geq 2600 \mathrm{~cm}^{2} / \mathrm{g}$ \\
\hline \multicolumn{2}{|l|}{ Finura $(\# 200)(\%)$} & 1,70 & $\leq 12,0 \%$ \\
\hline \multicolumn{2}{|l|}{ Finura $(\# 325)(\%)$} & 6,70 & - \\
\hline \multirow[b]{2}{*}{ Tempo de pega $(\mathrm{min})$} & Início de pega & 168 & $\geq 60 \mathrm{~min}$ \\
\hline & Fim de pega & 226 & $\leq 600 \mathrm{~min}$ \\
\hline \multirow[b]{3}{*}{ Resistência à compressão (Mpa) } & 3 dias & 27,4 & $\geq 10,0 \mathrm{Mpa}$ \\
\hline & 7 dias & 31,8 & $\geq 20,0 \mathrm{MPa}$ \\
\hline & 28 dias & 37,1 & $\geq 32,0 \mathrm{MPa}$ \\
\hline
\end{tabular}

A Tabela 2 apresenta um resumo das normas utilizadas para caracterização dos agregados, bem como os resultados obtidos com os agregados analisados.

Tabela 2: Características dos agregados miúdos e graúdos utilizados na pesquisa.

\begin{tabular}{|c|c|c|c|c|}
\hline ENSAIO & NORMA & AREIA & BRITA 0 & BRITA 1 \\
\hline Granulometria & ABNT NM 248:2003 [26] & $\begin{array}{l}\mathrm{DMC}=2,36 \mathrm{~mm} \\
\mathrm{MF}=2,52\end{array}$ & $\begin{array}{l}\mathrm{DMC}=12,5 \mathrm{~mm} \\
\mathrm{MF}=5,99\end{array}$ & $\begin{array}{l}\mathrm{DMC}=19 \mathrm{~mm} \\
\mathrm{MF}=6,84\end{array}$ \\
\hline Massa unitária $\left(\mathrm{g} / \mathrm{cm}^{3}\right)$ & ABNT NBR NM 45:2006 [27] & 1,5 & 1,41 & 1,37 \\
\hline Absorção de água (\%) & & 0,2 & 0,89 & 0,94 \\
\hline Massa específica $\left(\mathrm{g} / \mathrm{cm}^{3}\right)$ & ABNT NBR NM 52:2009 [28] & 2,64 & 2,61 & 2,61 \\
\hline Material pulverulento (\%) & ABNT NBR NM 46:2003 [29] & 1,7 & 0,29 & 0,9 \\
\hline
\end{tabular}

$\mathrm{O}$ aditivo utilizado foi do tipo superplastificante, composto por policarboxilatos, à base de naftaleno sulfonato. Possui cor amarelada, $\mathrm{pH}$ entre 8,50 e 10,40 e uma massa específica em torno de 1,05 a $1,10 \mathrm{~g} / \mathrm{cm}^{3}$. A fibra de polipropileno apresenta absorção zero, à prova de álcalis, ponto de ignição $404^{\circ} \mathrm{C}$, ponto de fusão $160^{\circ} \mathrm{C}$, massa específica 0,91 e baixa condutividade elétrica e térmica.

\subsection{Métodos de pesquisa}

Primeiramente, deu-se início com o preparo do concreto e moldagem dos corpos de prova cilíndricos de dimensões $10 \mathrm{~cm}$ de diâmetro e $20 \mathrm{~cm}$ de altura, os quais foram submetidos à cura por 28 dias. Os traços utilizados são apresentados nas Tabela 3, juntamente com os materiais utilizados e suas quantidades. Para a consistência do concreto foi adotado um slump de $8 \pm 2$ mm, obtido de acordo com a NBR NM 67 [30].

Tabela 3: Consumo de materiais por $\mathrm{m}^{3}$ de concreto, em $\mathrm{kg}$.

\begin{tabular}{l|l|l|l|l|l|l|l|l}
\hline TRAÇO & $\begin{array}{l}\mathbf{f}_{\text {ck }} \\
\text { (Mpa) }\end{array}$ & CIMENTO & AREIA & $\begin{array}{l}\text { BRITA } \\
\text { 13mm }\end{array}$ & $\begin{array}{l}\text { BRITA } \\
\text { 19mm }\end{array}$ & $\begin{array}{l}\text { ADITIVO } \\
\text { (\%) }\end{array}$ & $\begin{array}{l}\text { FIBRA DE POLI- } \\
\text { PROPILENO }\end{array}$ & $\begin{array}{l}\text { FATOR } \\
\text { a/c }\end{array}$ \\
\hline T20 & 20,0 & 278 & 860 & 277 & 694 & 0,23 & - & 0,65 \\
\hline $\mathbf{T 3 0}$ & 30,0 & 332 & 794 & 264 & 703 & 0,72 & - & 0,54 \\
\hline $\mathbf{T 3 0 + 1 , 2}$ & 30,0 & 332 & 794 & 264 & 703 & 0,76 & $1,20 \mathrm{~kg} / \mathrm{m}^{3}$ & 0,54 \\
\hline $\mathbf{T 3 0 + 1 , 8}$ & 30,0 & 332 & 794 & 264 & 703 & 0,85 & $1,80 \mathrm{~kg} / \mathrm{m}^{3}$ & 0,54 \\
\hline $\mathbf{T 3 0 + 2 , 4}$ & 30,0 & 332 & 794 & 264 & 703 & 0,94 & $2,40 \mathrm{~kg} / \mathrm{m}^{3}$ & 0,54 \\
\hline $\mathbf{T 4 0}$ & 40,0 & 435 & 710 & 266 & 709 & 0,80 & - & 0,41 \\
\hline $\mathbf{T 5 0}$ & 50,0 & 542 & 997,6 & - & 673,4 & 1,02 & - & 0,33 \\
\hline
\end{tabular}

Em seguida, os corpos de prova foram secos em estufa por 24 horas a $105{ }^{\circ} \mathrm{C} \pm 5{ }^{\circ} \mathrm{C}$, posteriormente à secagem, foi realizado a pesagem dos corpos de prova e realizado o ensaio de ultrassom para determinar a velocidade de propagação ultrassônica. Logo após, os corpos de prova foram submetidos às temperaturas de $200{ }^{\circ} \mathrm{C}, 400{ }^{\circ} \mathrm{C}, 600{ }^{\circ} \mathrm{C}$ e $800{ }^{\circ} \mathrm{C}$, à uma taxa de aquecimento conforme ilustrado na Figura 1, utilizando-se 
um forno elétrico, com capacidade de temperatura máxima de $1340{ }^{\circ} \mathrm{C}$, isolamento térmico através de placas de silicato de cálcio e equipado com um sistema digital de controle de temperatura e tempo. A temperatura adotada como referência (REF.) foi a temperatura ambiente, com medida em torno de $28{ }^{\circ} \mathrm{C}$.

Para o tempo requerido de resistência ao fogo foi adotado o período médio de 30 minutos, conforme definido na ABNT NBR 14432:2001 [31] para ocupações residenciais de altura até 12 metros de altura. Os corpos de prova foram expostos ao calor durante o processo de aquecimento do forno até se alcançar a temperatura desejada para estudo, e em seguida, realizado a marcação do tempo de exposição. A Tabela 4 apresenta uma divisão dos parâmetros e variáveis analisados para a realização da pesquisa, bem como a quantidade de corpos de provas utilizados em cada ensaio.

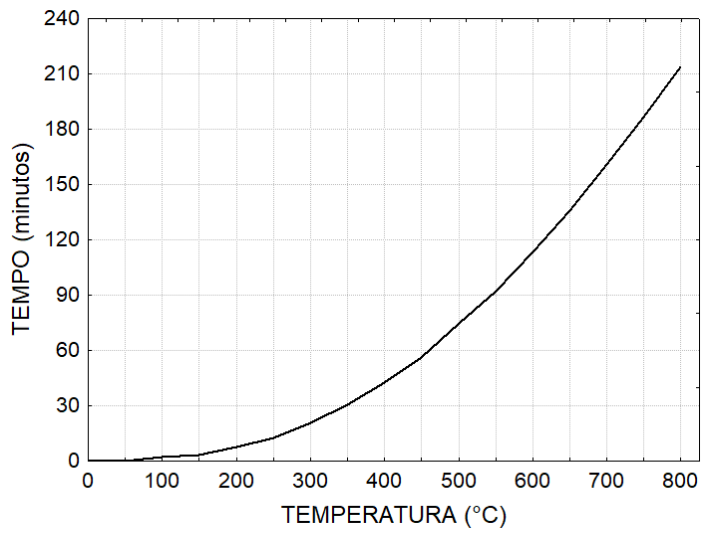

Figura 1: Taxa de aquecimento do forno utilizado.

Tabela 4: Divisão dos parâmetros analisados.

\begin{tabular}{|c|c|c|c|c|c|}
\hline PARÂMETRO & TRAÇOS & TIPO DE RESFRIAMENTO & ENSAIOS & $\begin{array}{l}\text { TEMPO DE } \\
\text { FORNO }\end{array}$ & $\begin{array}{l}N^{\circ} \mathrm{DE} \\
\text { CP's }\end{array}$ \\
\hline Módulo de elasticidade & $\mathrm{T} 30$ & Natural & Módulo de elasticidade & $30 \mathrm{~min}$ & 15 \\
\hline Tipo de resfriamento & $\mathrm{T} 30$ & $\begin{array}{l}\text { Brusco (imerso em água) } \\
\text { Natural }\end{array}$ & Resistência à compressão & $30 \mathrm{~min}$ & 30 \\
\hline Tempo de exposição & $\mathrm{T} 30$ & Natural & Resistência à compressão & $\begin{array}{l}15 \mathrm{~min} \\
30 \mathrm{~min} \\
60 \mathrm{~min}\end{array}$ & $\begin{array}{l}15 \\
15 \\
15 \\
\end{array}$ \\
\hline$f_{c k}$ do concreto & $\begin{array}{l}\text { T20 } \\
\text { T30 } \\
\text { T40 } \\
\text { T50 }\end{array}$ & Natural & $\begin{array}{l}\text { Resistência à compressão } \\
\text { Perda de massa } \\
\text { VPU }\end{array}$ & $30 \mathrm{~min}$ & 60 \\
\hline $\begin{array}{l}\text { Teor de fibra de poli- } \\
\text { propileno }\end{array}$ & $\begin{array}{l}\mathrm{T} 30+1,2 \\
\mathrm{~T} 30+1,2 \\
\mathrm{~T} 30+1,2\end{array}$ & Natural & $\begin{array}{l}\text { Resistência à compressão } \\
\text { VPU }\end{array}$ & $30 \mathrm{~min}$ & 45 \\
\hline
\end{tabular}

Por fim, após a coleta dos resultados experimentais, foi realizada uma Análise de Variância (ANOVA) e utilizado o método de comparação das médias através do Teste de Tukey por meio do software Statistica 7.0 para análise dos dados, em todos os parâmetros analisados. Como representação gráfica do Teste de Tukey foram utilizadas linhas horizontais acima das barras nos gráficos.

\section{RESULTADOS E DISCUSSÕES}

\subsection{Módulo de elasticidade}

O módulo de elasticidade é um parâmetro de extrema importância no dimensionamento de estruturas de concreto armado pois é fundamental quando se trata de analisar as suas deformações, o que torna necessá- 
rio identificar esta variável nos projetos estruturais. Foi analisado o comportamento do módulo de elasticidade do concreto quando exposto à altas temperaturas. A ANOVA realizada demonstra que a temperatura influencia significativamente o comportamento do módulo de elasticidade. Com a realização do teste de comparação de médias de Tukey, foi identificado que não há diferença significativa entre as médias do módulo de elasticidade obtidos para os concretos de referência e o aquecido a $200{ }^{\circ} \mathrm{C}$. As médias dos demais diferem entre si, conforme Figura 2.

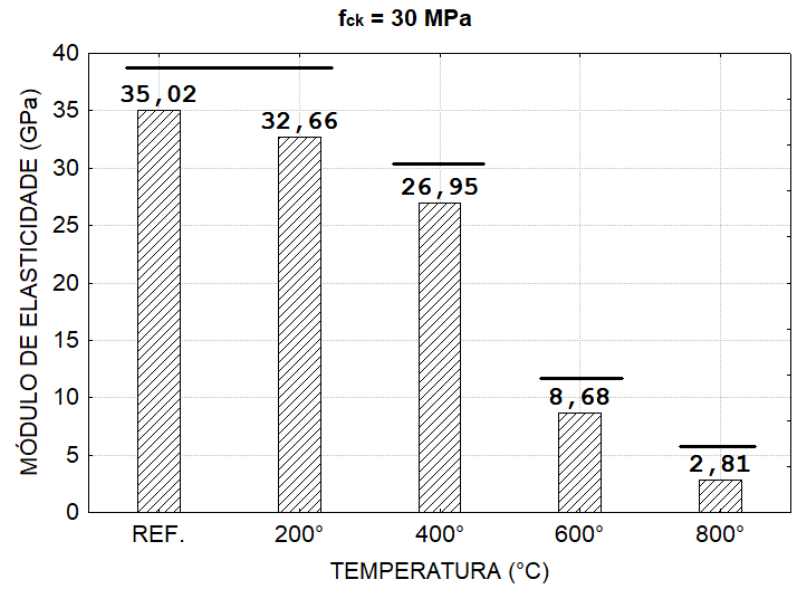

Figura 2: Desempenho do módulo de elasticidade conforme aumento da temperatura.

Percebe-se que em $200{ }^{\circ} \mathrm{C}$ o módulo de elasticidade é pouco afetado, com fator de redução na ordem de 0,93 . Em $400^{\circ} \mathrm{C}$ tem-se um fator de redução de 0,77 , o qual a partir desta temperatura o módulo é drasticamente afetado, com fatores de redução na ordem de 0,25 e 0,08 para as temperaturas de $600{ }^{\circ} \mathrm{C}$ e $800{ }^{\circ} \mathrm{C}$, respectivamente. Valores similares foram encontrados por LIMA [32], que obteve valores de fator de redução na ordem de $0,93 \%, 0,77 \%$ e $0,25 \%$ para as temperaturas de $200{ }^{\circ} \mathrm{C}, 400{ }^{\circ} \mathrm{C}$ e $600{ }^{\circ} \mathrm{C}$, respectivamente.

\subsection{Tempo de exposição e tipo de resfriamento}

Em relação a influência do tempo de exposição a altas temperaturas, foi investigado a resistência à compressão residual. Foram analisados os tempos de 15, 30 e 60 minutos. Com a ANOVA foi identificado que o tempo de exposição influencia significativamente a resistência à compressão do concreto. O teste de Tukey constatou que apenas há diferença significativa entre as médias para os tempos de exposição de 15 e 60 minutos, sendo essa diferença apenas para a temperatura de $600{ }^{\circ} \mathrm{C}$ (Figura 3).

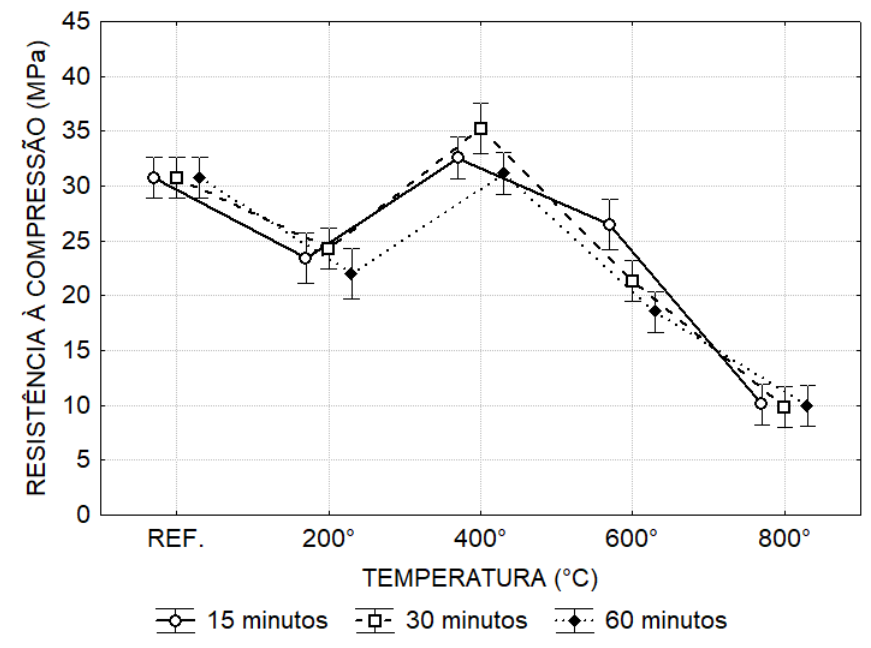

Figura 3: Variação da resistência à compressão em relação ao tempo de exposição e aumento de temperatura. 
Em seguida, foi verificado a influência do tipo de resfriamento, natural (ao ar livre) e brusco (submerso em água imediatamente após a retirada do forno), na resistência à compressão residual do concreto. Apesar do resfriamento brusco apresentar menores valores de resistência, a ANOVA identificou que não há influência significativa do tipo de resfriamento na resistência do concreto. A Figura 4 ilustra o comportamento da resistência à compressão com o aumento da temperatura para os tipos de resfriamento.

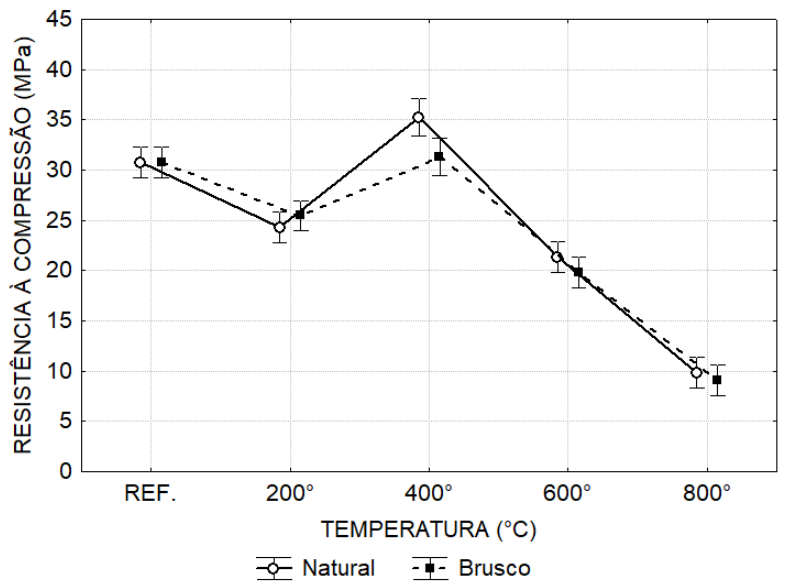

Figura 4: Comportamento da resistência à compressão conforme o tipo de resfriamento em diferentes temperaturas.

\subsection{Influência do $f_{c k}$}

Em relação a influência do $\mathrm{f}_{\mathrm{ck}}$, foi investigado o comportamento da resistência à compressão, perda de massa e velocidade de propagação ultrassônica, de concretos expostos a elevadas temperaturas. A ANOVA identificou que a exposição do concreto a altas temperaturas influencia significativamente a sua resistência residual. Com a realização do teste de comparação de médias de Tukey constatou-se que há diferenças significativas entre as médias dos concretos produzidos, para todos os valores de $\mathrm{f}_{\mathrm{ck}}$ estudados.

Conforme apresentado na Figura 5, para os concretos de 20, 30 e $40 \mathrm{MPa}$ não há diferença significativa entre as médias das resistência até $400{ }^{\circ} \mathrm{C}$. Para o concreto de $50 \mathrm{MPa}$ até $200{ }^{\circ} \mathrm{C}$ não há diferença entre as médias, porém em $400{ }^{\circ} \mathrm{C}$ ocorre um aumento de resistência, se comparado com o de referência. Fato similar de aumento de resistência com o aumento da temperatura foi encontrado por diversos autores na literatura. [1, $25,33,34,35,36,37,38,39,40,41,42,43,44]$

Conforme HUO et al. [43] foi observado que quando a temperatura era inferior, porém próxima a 400 ${ }^{\circ} \mathrm{C}$ a reidratação de partículas de cimento continuou a gerar novos $\mathrm{Ca}(\mathrm{OH})_{2}(\mathrm{CH})$ e $(\mathrm{C}-\mathrm{S}-\mathrm{H})$. Para MA et al. e HAGER [1,40] a hidratação dos grãos de cimento não hidratados é melhorada devido a uma condição de autoclave interna como resultado da alta temperatura e evaporação da água. Já SAVVA et al. [36] explicam que para o material pozolânico, formou-se um gel adicional de tobermorite como resultado da reação pozolânica de $\mathrm{Ca}(\mathrm{OH})_{2}$. POON et al. [33] ainda complementa afirmando que esse aumento é mais pronunciado no concreto com maior porcentagem de partículas não hidratadas devido à sua estrutura densa.

A partir de $600^{\circ} \mathrm{C}$ tem-se uma acentuada redução na resistencia à compressão para todos os $\mathrm{f}_{\mathrm{ck}}$ investigado. SOUZA e MORENO JR [45] explicam que nesta temperatura, o concreto perde não só a água livre, mas também a água contida no gel do cimento, provocando, como consequência, um alto grau de fissuração superficial. Os agregados se expandem dando lugar a tensões internas que prejudicam a resistência à compressão do concreto, o que corroboram com os resultados obtidos. 

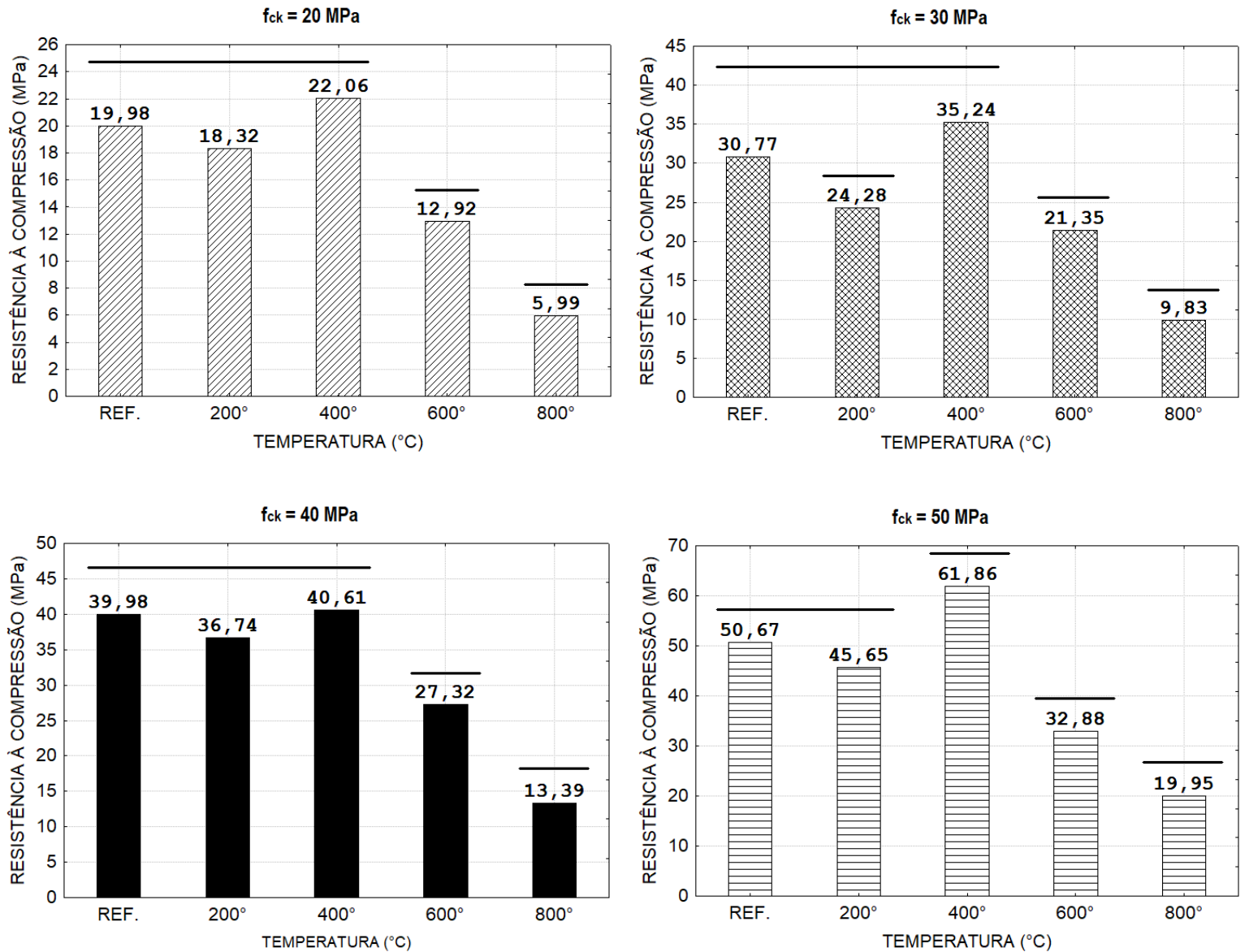

Figura 5: Análise comparativa do desempenho mecânico entre os tipos de $f_{c k}$ analisados em diferentes temperaturas.

Quanto à perda de massa dos concretos com diferentes $\mathrm{f}_{\mathrm{ck}}$, o teste de TUKEY identificou que a diferença entre as médias não é significativa entre os concretos e 20, 30 e $40 \mathrm{MPa}$, sendo divergente apenas para o concreto de $50 \mathrm{MPa}$. A Figura 6 apresenta os valores de perda de massa para cada concreto, conforme a temperatura.

Constata-se que a perda de massa do concreto aumenta com o aumento da temperatura. A perda de massa está associada a evaporação da água livre ou fisicamente ligada. CASTRO et al. [46], explicam que na primeira hora, a taxa de evaporação é reduzida já que o calor só remove a umidade próxima à superfície da amostra.

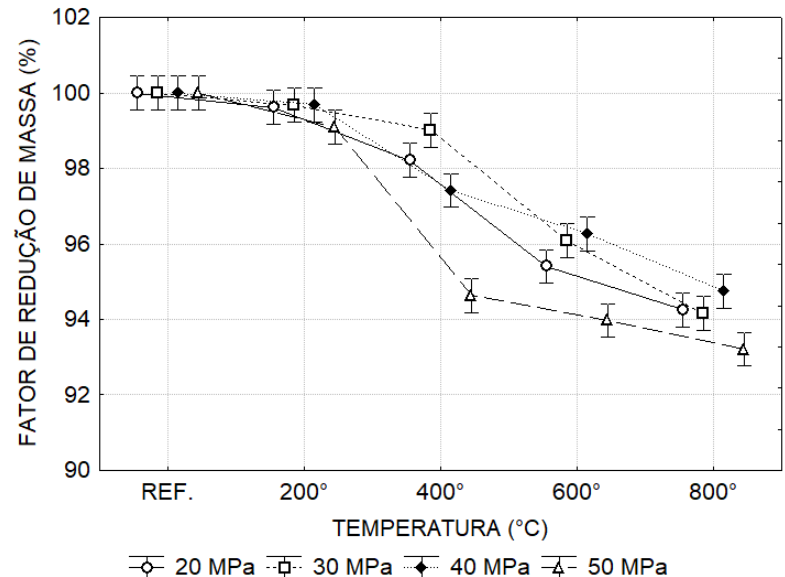

Figura 6: Influência dos diferentes tipos de $\mathrm{f}_{\text {ck }}$ na redução de massa do corpo de prova exposto a elevadas temperaturas. 
Quanto à redução da velocidade ultrassônica do concreto, percebe-se uma redução da velocidade de propagação ultrassônica no concreto com o aumento da temperatura. A ANOVA demonstrou que o $f_{\text {ck }}$, bem como a interação entre temperatura- $f_{c k}$ influencia significativamente na redução da velocidade. Com a realização do teste de TUKEY para o tipo de $\mathrm{f}_{\mathrm{ck}}$ foi verificado que o valor das médias do fator de redução da velocidade ultrassônica é diferente para cada tipo, sendo o maior fator de redução para o concreto de $20 \mathrm{MPa}$. Foi constatado que até $200^{\circ} \mathrm{C}$ não ocorre diferença significativa na VPU, o que começa a ocorrer a partir de $400{ }^{\circ} \mathrm{C}$ (Figura 7).
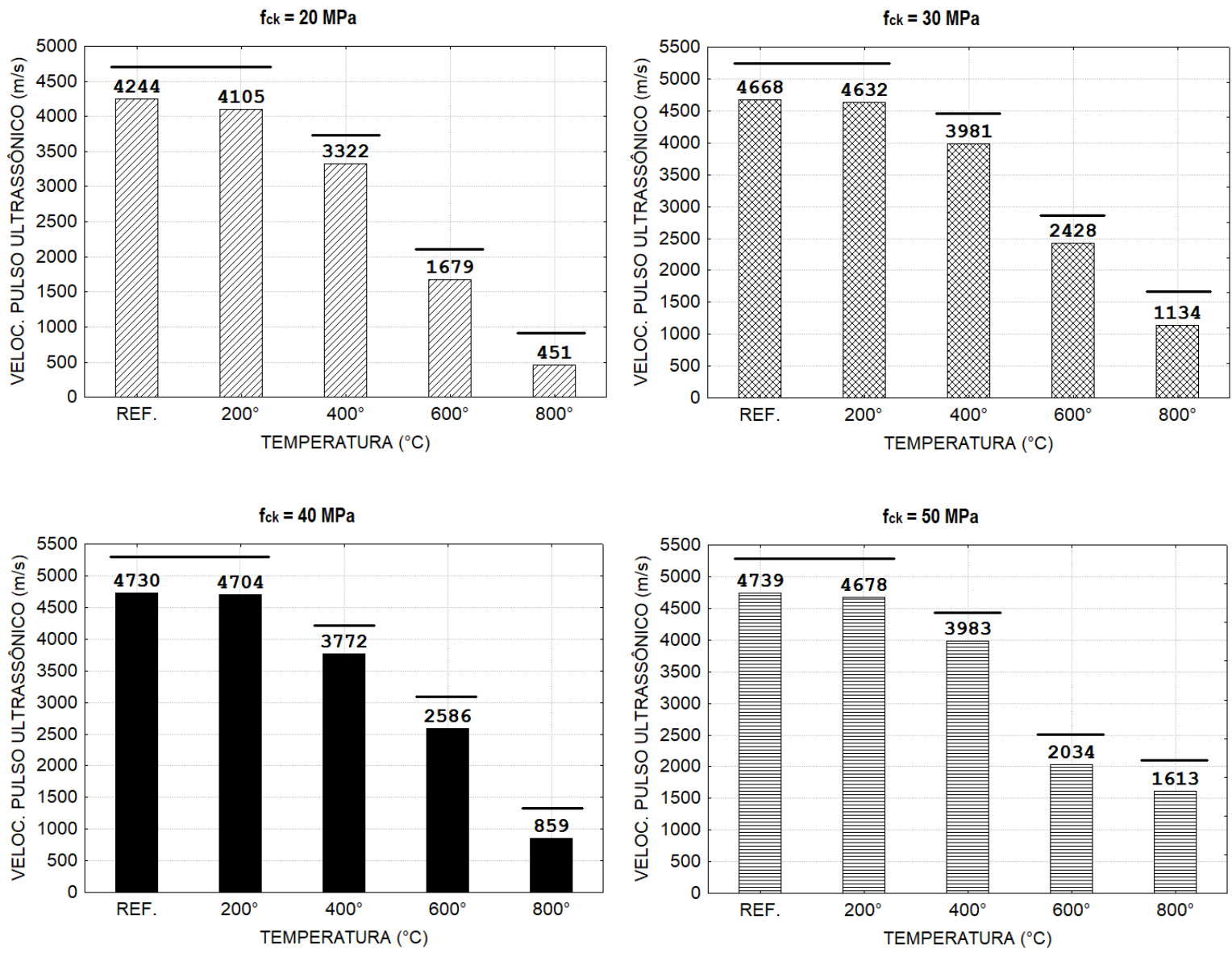

Figura 7: Comportamento da velocidade de pulso ultrassônico de acordo com a mudança de temperatura e $\mathrm{f}_{\mathrm{ck}}$.

Constata-se que a partir de $400^{\circ} \mathrm{C}$ tem-se uma redução significativa na velocidade, sendo mais acentuada a $600^{\circ} \mathrm{C}$, o que pode ser justificado pelo maior grau de deterioração do concreto e maior incidência de fissuras, que conforme SOUZA e MORENO JR [45], ocorre a partir de $600^{\circ} \mathrm{C}$. Em consoante, percebe-se que o concreto com menor $f_{\text {ck }}$ apresenta maiores valores de redução da VPU, o que provavelmente se deva a uma maior quantidade de poros e fissuras no concreto quando submetido a elevadas temperaturas.

A coloração da superfície externa dos concretos é observada na Figura 8. Conforme GEORGALI e TSAKIRIDIS [6], as cores observadas em concretos submetidos a elevadas temperaturas seriam tons de rosa a avermelhado entre $300{ }^{\circ} \mathrm{C}$ a $600{ }^{\circ} \mathrm{C}$, tom de cinza a amarelado $600{ }^{\circ} \mathrm{C}$ até cerca de $900{ }^{\circ} \mathrm{C}$ e a partir de 900 ${ }^{\circ} \mathrm{C}$ um tom amarelado escuro. 

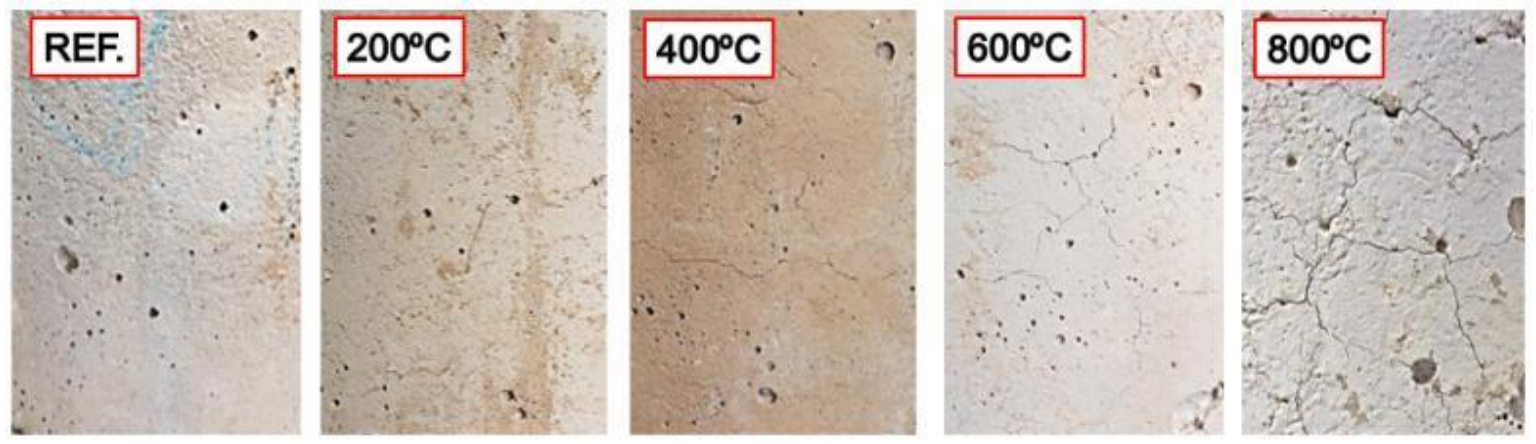

Figura 8: Variação da coloração da superfície externa dos corpos de prova de concreto após exposição a determinadas temperaturas.

\subsection{Teor de fibras de polipropileno}

Quanto a influência da adição de fibra de polipropileno para concretos expostos a elevadas temperaturas, a ANOVA identificou que o teor de fibras e a interação temperatura-teor de fibras influenciam significativamente a resistência à compressão do concreto após a exposição a elevadas temperaturas. A Figura 9 apresenta o comportamento dos concretos com os três teores de fibra quando aquecidos.

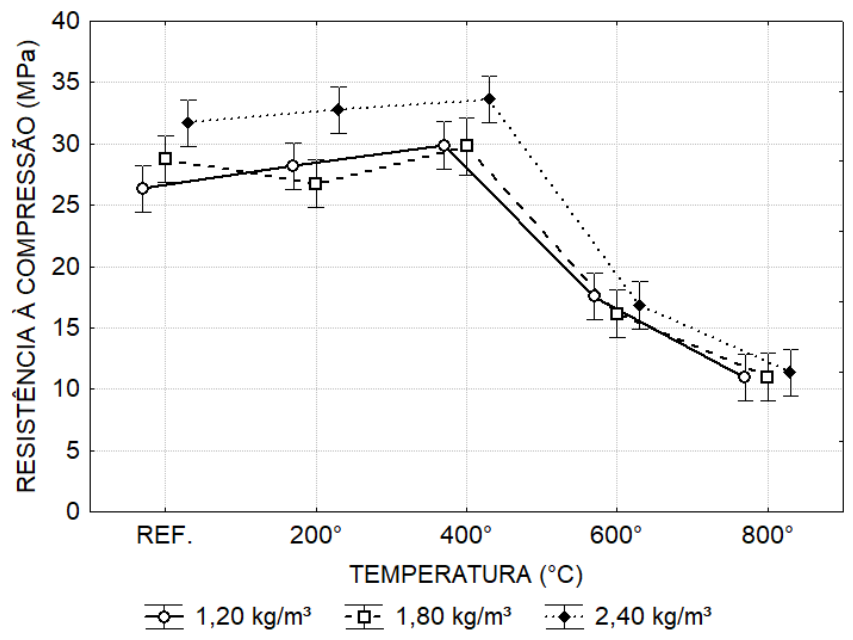

Figura 9: Influência da temperatura na resistência à compressão para traços contendo diferentes teores de fibra.

Percebe-se na Figura 9 que até a temperatura de $400{ }^{\circ} \mathrm{C}$ o concreto com teor de fibra de $2,40 \mathrm{~kg} / \mathrm{m}^{3}$ apresenta os maiores valores de resistência. Já a partir de $600{ }^{\circ} \mathrm{C}$ o teste de TUKEY afirma que não há diferença significativa entre as médias das resistências para os três teores de adição de fibra, ou seja, a resistência à compressão residual do concreto após exposto a elevadas temperaturas não foi influenciada pelos teores de adição de fibra a partir de $600{ }^{\circ} \mathrm{C}$.

Constata-se com os resultados do teste de TUKEY que não há diferenças significativas entre as médias obtidas para os concretos com teores de $1,20 \mathrm{~kg} / \mathrm{m}^{3}$ e $1,80 \mathrm{~kg} / \mathrm{m}^{3}$, sendo apenas para o valor de 2,40 $\mathrm{kg} / \mathrm{m}^{3}$ que apresenta diferença significativa.

A Figura 10 apresenta comparação das médias por meio do teste de TUKEY de acordo com a temperatura para cada teor individualmente. Pode ser observado que para todos os teores de adição de fibra não há diferença significativa entre as médias até $400{ }^{\circ} \mathrm{C}$, a partir de $600{ }^{\circ} \mathrm{C}$ já ocorre uma redução significativa. Já para os teores de $1,20 \mathrm{~kg} / \mathrm{m}^{3}$ e $1,80 \mathrm{~kg} / \mathrm{m}^{3}$ não há diferenças significativas entre as médias para nenhuma das temperaturas. 
TEOR = SEM FIBRAS

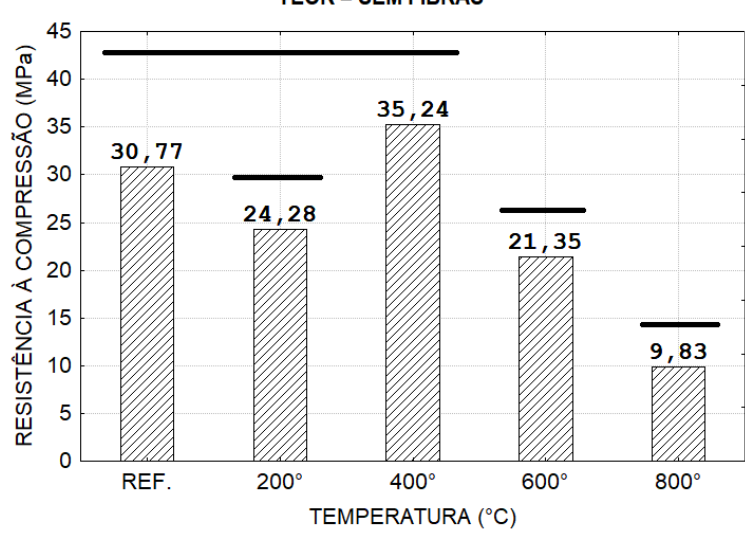

$\mathrm{TEOR}=1,80 \mathrm{~kg} / \mathrm{m}^{3}$

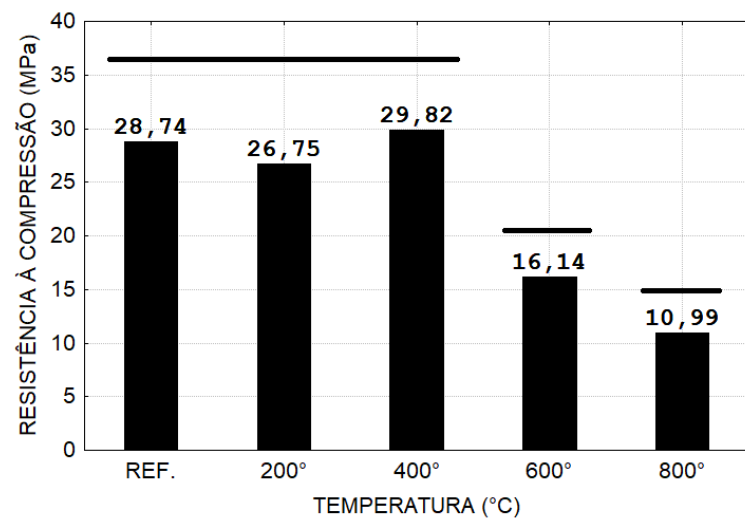

$\mathrm{TEOR}=1,20 \mathrm{~kg} / \mathrm{m}^{3}$

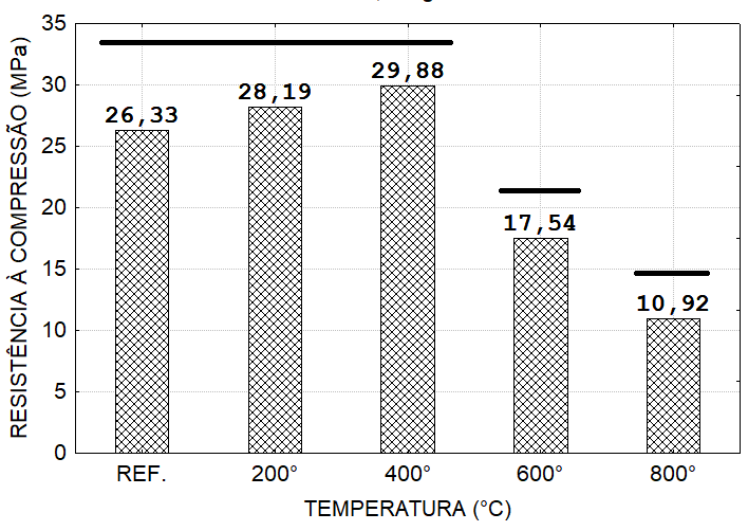

TEOR $=2,40 \mathrm{~kg} / \mathrm{m}^{3}$

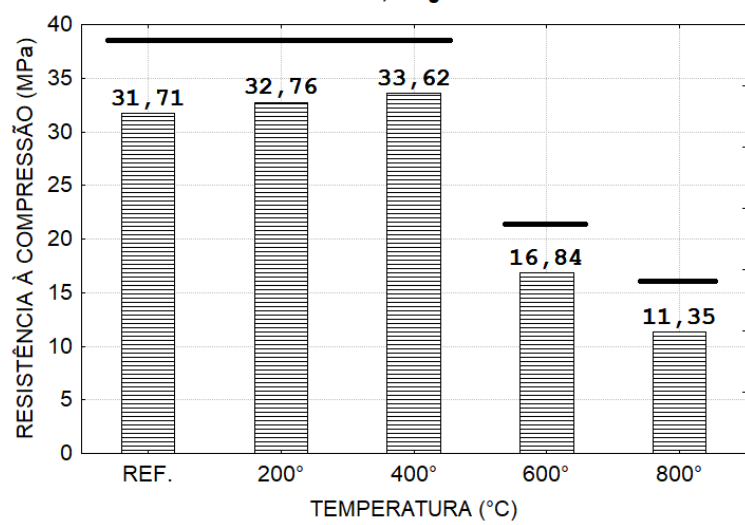

Figura 10: Análise comparativa do desempenho mecânico entre os traços com diferentes teores de fibra de polipropileno em diferentes temperaturas.

Em relação à redução da velocidade de pulso ultrassônico no concreto, a ANOVA demonstrou que o teor de adição de fibras de polipropileno influencia significativamente a redução da velocidade, sendo o teor de $1,80 \mathrm{~kg} / \mathrm{m}^{3}$ que apresenta o menor fator de redução. A interação entre temperatura-teor de fibras também influencia significativamente a redução da velocidade de propagação do ultrassom no concreto, conforme a ANOVA. O teste de TUKEY para o efeito combinado de temperatura-teor de fibras é demonstrado na Figura 11.

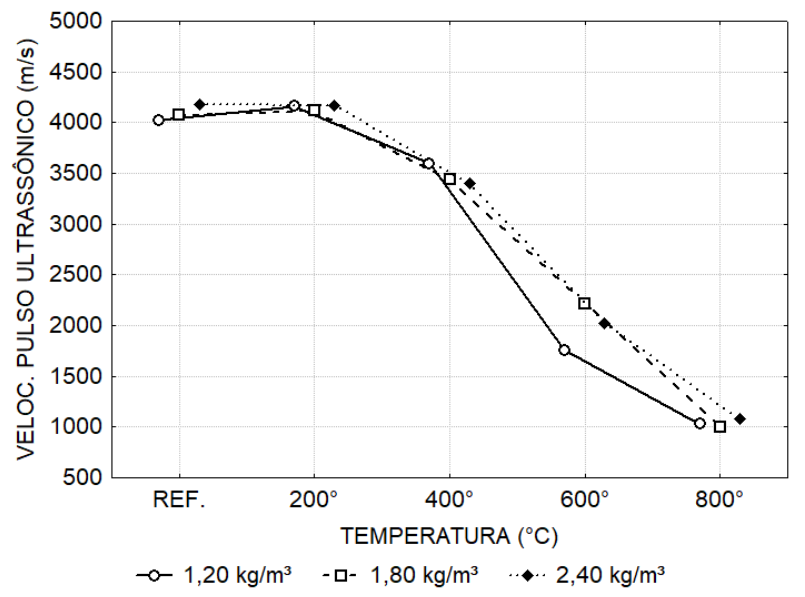

Figura 11: Influência dos diferentes teores de fibra de polipropileno na velocidade de pulso ultrassônico do concreto quando exposto a elevadas temperaturas. 
A adição de fibra de polipropileno ajudou a prevenir o surgimento do fenômeno spalling. Até $600{ }^{\circ} \mathrm{C}$ não ocorreu spalling do concreto para nenhum teor de adição, enquanto que esse lascamento na superfície do concreto de referência já ocorria, sendo mais intensificado nos concretos de $50 \mathrm{MPa}$, devido à sua menor permeabilidade e estrutura mais densa.

Para a temperatura de $800{ }^{\circ} \mathrm{C}$ ocorreu o spalling para todos os concretos com adição de fibras de polipropileno, sendo menos intensificado no traço com maior teor de adição $\left(2,40 \mathrm{~kg} / \mathrm{m}^{3}\right)$, conforme Figura 12 , onde podem ser observadas imagens da superfície dos corpos de prova imediatamente após a exposição à esta temperatura. Foi escolhida a região mais crítica do corpo de prova, ou seja, com a maior presença de spalling, em uma área superficial de 3,5 cm de largura por 5,0 $\mathrm{cm}$ de altura.
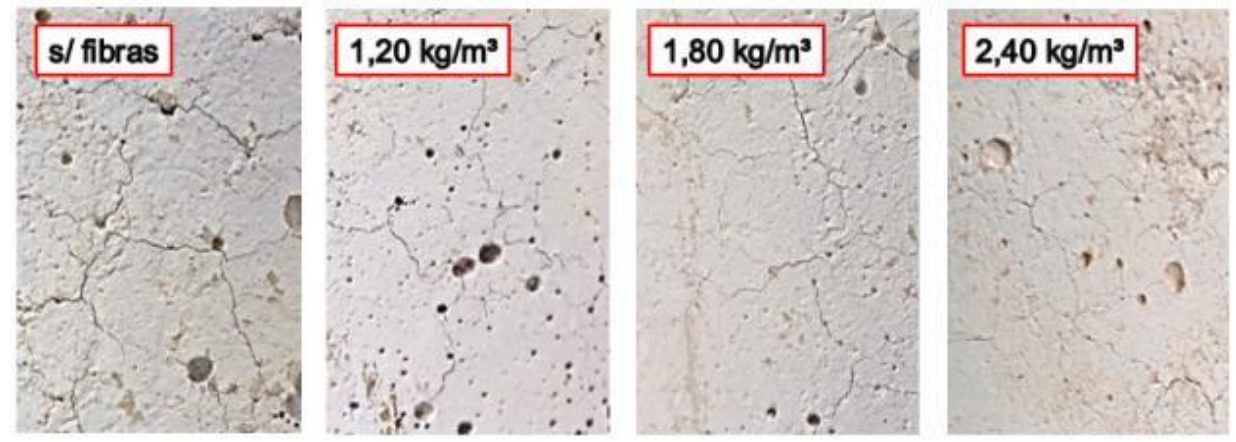

Figura 12: Diminuição da ocorrência do spalling no concreto à medida que se aumenta o teor de fibra de polipropileno, em concretos expostos à temperatura de $800{ }^{\circ} \mathrm{C}$ por 30 minutos.

\section{CONCLUSÕES}

O módulo de elasticidade é uma das propriedades mais afetadas com o aumento da temperatura. Foi observado que até $200{ }^{\circ} \mathrm{C}$ não há diferença significativa em comparação às médias do resultados obtidos para os corpos de prova de referência, sendo a partir de $400{ }^{\circ} \mathrm{C}$ quando se inicia a redução considerável do módulo de elasticidade do concreto, chegando a atingir uma redução de $92 \%$ para o concreto submetido a temperatura de $800{ }^{\circ} \mathrm{C}$.

O tempo de exposição causa influência na resistência residual do concreto, sendo o tempo de 60 minutos, o que apresentou as maiores perdas de resistência. Quanto ao tipo de resfriamento, não houve influência na resistência residual do concreto. Dessa forma, o concreto ao ser resfriado ao ar livre de forma natural, ou bruscamente com uso de água, não ocasiona alterações nas propriedades mecânicas.

Quanto aos resultados encontrados a partir da análise do diferentes tipos de $\mathrm{f}_{\mathrm{ck}}$, foi possível avaliar que até $400{ }^{\circ} \mathrm{C}$, com exceção do concreto com resistência de $50 \mathrm{MPa}$, não há diferença significativa entre as médias da resistência residual do concreto, acima desta temperatura o concreto começa a sofrer redução significativa na resistência e apresentar um alto grau de fissuração superficial, ocasionados principalmente pela perda de água livre e contida no gel do cimento e à tensões internas oriundas da expansão dos agregados.

Constatou-se que a perda de massa do concreto e, consequentemente, a redução da velocidade de pulso ultrassônico aumentam com o acréscimo da temperatura, por estarem associada à evaporação da água livre ou fisicamente ligada. Para a perda de massa foi observado um fator de redução em média de $6 \%$ nas maiores temperaturas estudadas, sendo essa perda mais acentuada para o traço de $50 \mathrm{MPa}$. Para a VPU, percebeu-se que sua redução começa a ser significativa a partir da temperatura de $400{ }^{\circ} \mathrm{C}$, sendo o maior fator de redução para o concreto de $20 \mathrm{MPa}$.

O fenômeno do spalling com a adição de fibras de polipropileno foi prevenido até a temperatura de exposição de $600{ }^{\circ} \mathrm{C}$. Acima desta temperatura a adição atuou apenas na minimização desse fenômeno, pois todos os concretos com fibras apresentaram spalling, fato menos intenso no concreto com maior teor de adição. Vale ressaltar que a resistência residual do concreto não é afetada com a adição de fibras quando o concreto é submetido a elevadas temperaturas.

Os resultados obtidos demonstram a importância das normas regulamentadoras que apresentam valores de redução para o módulo de elasticidade e para a resistência a compressão para o concreto após submetidas a situação de incêndios. Com isso, o engenheiro pode mensurar os danos causados pelo fogo nas estruturas de concreto e assim, estabelecer parâmetros para a construção de novos empreendimentos, bem como critérios para reforço e recuperação de estruturas que passaram por sinistros. 


\section{AGRADECIMENTOS}

Os autores agradecem ao Programa de Pós-Graduação em Engenharia Civil - PEC, ao Departamento de Engenharia Metalúrgica e de Materiais, e ao Laboratório de Materiais de Construção Civil da Universidade Federal do Ceará - UFC. O presente trabalho foi realizado com apoio da Coordenação de Aperfeiçoamento de Pessoal de Nível Superior - Brasil (CAPES) - Código de Financiamento 001.

\section{BIBLIOGRAFIA}

[1] MA, Q. GUO, R. ZHAO, Z., et al., "Mechanical properties of concrete at high temperature - A review", Construction and Building Materials, v. 93 pp. 371-383, set. 2015.

[2] SOUZA, A. A. A., MORENO JR, A. L. "The effect of high temperatures on concrete compression strength, tensile strength and deformation modulus", Ibracon Structures and Materials Journal, v.3, n.4 pp.432-448, dec.2010

[3] LAU, A., ANSON, M. "Effect of high temperatures on high performance steel fiber reinforced concrete", Cement and Concrete Research. v. 36, n. 9, pp. 1698-1707, set. 2006.

[4] KODUR, V. "Properties of Concrete at Elevated Temperatures". ISRN Civil Engineering. Vol 2014, pp 115 , mar. 2014

[5] POON, C., AZHAR, S., ANSON, M., et al., "Strength and durability recovery of fire-damaged concrete after post fire curing", Cement and Concrete Research. v. 31, pp. 1307-1318, set. 2001.

[6] GEORGALI, B., TSAKIRIDIS, P. E. "Microstructure of fire-damaged concrete. A case study", Cement \& concrete composites. v.27, pp. 255-259, Fev. 2005.

[7] ARIOZ, O. "Effects of elevated temperatures on properties of concrete", Fire Safety Journal, v.42 pp. 516-522, Nov 2007.

[8] YANG, H., LIN, Y., HSIAO, C., et al., "Evaluating residual compressive strength of concrete at elevated temperatures using ultrasonic pulse velocity", Fire Safety Journal, v.44, pp.121-130, Jan 2009.

[9] HADDAD, R.H., SHANNIS, L.G. "Post-fire behavior of bond between high strength pozzolanic concrete and reinforcing steel", Construction and Building Materials, v.18, pp. 425-435, Jul. 2004.

[10] XIAO, J., G. KÖNIG, G. "Study on concrete at high temperature in China - an overview", Fire Safety Journal, v. 39, pp. 89-103, Fev. 2004.

[11] HUSEM, M. "The effects of high temperature on compressive and flexural strengths of ordinary and high-performance concrete", Fire Safety Journal, v.41, pp. 155-163, Mar. 2006.

[12] YICHING, L., CHIAMEN, H., HSUANCHIH, Y., et al., "The effect of post-fire-curing on strengthvelocity relationship for nondestructive assessment of fire-damaged concrete strength", Fire Safety Journal. v.46, n. 4 pp. 178-185, Maio 2011.

[13] PARK, S., YIM, H.J., KWAK, H. "Effects of post-fire curing conditions on the restoration of material properties of fire-damaged concrete", Construction and Building Materials. V.99, pp. 90-98, Nov. 2015.

[14] HERTZ K. D., SORENSEN, L. S. "Test method for spalling of fire exposed concrete". Fire Safety Journal. v. 40, n. 5 pp. 466-676, Jul. 2005.

[15] LEE, G., HAN, D., HAN, M.C., et al., "Combining polypropylene and nylon fibers to optimize fiber addition for spalling protection of high-strength concrete", Construction and building Materials. V.34, pp. 313-320, Set. 2012.

[16] SIDERIS, K. K., MANITA, P. "Residual mechanical characteristics and spalling resistance of for reinforced self-compacting concretes exposed to elevated temperatures", Construction and Building Materials, v.41, pp. 296-302, Abr. 2013.

[17] YERMAK, N., PLIYA, P., BEAUCOUR, A., et al., "Influence of steel and/or polypropylene fibers on the behavior of concrete at high temperature: Spalling, transfer and mechanical properties", Construction and Building Materials, v.132, pp. 240-250, Fev. 2017.

[18] CHEN B, LIU J. "Residual strength of hybrid-fiber reinforced high strength concrete after exposure to high temperature", Cement and Concrete Research., v. 34, pp. 1065-1069, Jun. 2004.

[19] JANSSON, R., BOSTRÖM, L. "The influence of pressure in the pore system on fire spalling of concrete", Fire Technology. v. 46, n.1, pp. 217-230, Jan 2010.

[20] BANGI, M. R., HORIGUCHI, T. "Pore pressure development in hybrid fiber-reinforced high strength concrete at elevated temperatures", Cement and Concrete Research., v.41, n.11, pp. 1150-1156, Nov. 2011. 
[21] KARAHAN, O., ATIS C. D. "The durability properties of polypropylene fiber reinforced fly ash concrete”, Materials \& Design, v..32, n.2, pp. 1044-1049, Dez. 2011.

[22] XIONG, M. X., LIEW, J. Y. R. "Spalling behavior and residual resistance of fiber reinforced Ultra-High performance concrete after exposure to high temperatures", Materiales de Construcción , v. 65, n. 320, 2015.

[23] BEHNOOD, A., GHANDEHARI, M. "Comparison of compressive and splitting tensile strength of high-strength concrete without polypropylene fibers heated to high temperatures", Fire Safety Journal, v.44, n.8, pp. 1015-1022, Nov. 2009.

[24] POON, C.S., SHUI, Z.H., LAM, L. "Compressive behavior of fiber reinforced high-perfomance concrete subjected to elevated temperatures", Cement and Concrete Research, v. 34, n.12, pp. 2215-2222, Dez. 2004.

[25] AKCA, A. H., OZYURT, N., ZIHNIOGLU, O. "High performance concrete under elevated temperatures", Construction and Building Materials, v. 44, pp. 317-328, Jul. 2013.

[26] ASSOCIAÇÃO BRASILEIRA DE NORMAS TÉCNICAS. NBR NM 248: Agregados - Determinação da composição granulométrica. Rio de Janeiro, 2003.

[27] ASSOCIAÇÃO BRASILEIRA DE NORMAS TÉCNICAS. NBR NM 45: Determinação da massa unitária e do volume de vazios. Rio de Janeiro, 2006.

[28] ASSOCIAÇÃO BRASILEIRA DE NORMAS TÉCNICAS. NBR NM 52: Agregado miúdo - Determinação da massa específica e massa específica aparente. Rio de Janeiro, 2009.

[29] ASSOCIAÇÃO BRASILEIRA DE NORMAS TÉCNICAS. NBR NM 46: Agregados - Determinação do material fino que passa através da peneira 75 um, por lavagem. Rio de Janeiro, 2003.

[30] ASSOCIAÇÃO BRASILEIRA DE NORMAS TÉCNICAS. NBR NM 67: Concreto - Determinação da consistência pelo abatimento do tronco de cone. Rio de Janeiro, 1998.

[31] ASSOCIAÇÃO BRASILEIRA DE NORMAS TÉCNICAS. NBR 14432: Exigências de resistência ao fogo de elementos construtivos de edificações - Procedimento. Rio de Janeiro, 2001.

[32] LIMA, R. C. A. "Investigação do comportamento de concretos em elevadas temperaturas", Tese de D.Sc., Universidade Federal do Rio Grande do Sul. Porto Alegre, 2005.

[33] POON, C.S., AZHAR, S., ANSON, M., et al., "Comparison of the strength and durability performance of normal and high-strenght pozzolanic concretes at elevated temperatures", Cement and Concrete Research, v. 31, pp. 1291-1300, Set. 2001.

[34] XU, Y., WONG, Y. L., POON, C. S. ANSON, M. "Impact on high temperature on PFA concrete", Cement and Concrete Research. v.31, pp. 1065-1073, Jul. 2001.

[35] VODÁK, F. TRTÍK, K. KAPICKOVÁ, O. HOSKOVÁ, S. DEMO, P. "The effect of temperature on strength - porosity relationship for concrete", Construction and Building Materials, v.18, pp. 529-534, 2004.

[36] SAVVA A., MANITA P., SIDERIS K. K. "Influence of elevated temperatures on the mechanical properties of blended cement concretes prepared with limestone and siliceous aggregates", Cement and Concrete Composites, V.27, pp. 239-248, Fev. 2005.

[37] BIOLZI, L., CATTANEO, S. ROSATI, G. "Evaluating residual properties of thermally damaged concrete", Cement \& Concrete Composites. V.30, pp. 907-916, Nov. 2008.

[38] KIM, G., KIM, Y., LEE, T. "Mechanical properties of high-strength concrete subjected to high temperature by stressed test", Transactions of Nonferrous metals society of China, v.19, pp. 128-133, Set. 2009.

[39] CÜLFIK, M.S., ÖZTURAN, T. "Mechanical properties of normal and high strength concretes subjected to high temperatures and using image analysis to detect bond deteriorations", Construction and Building Materials, v.24, pp. 1486-1493, Ago. 2010.

[40] HAGER, I. "Behavior of cement concrete at high temperature", Bulletin of the polish academy of sciences Technical sciences, v. 61, pp. 145-154, Maio 2013.

[41] RASHAD, A.M. "An investigation of high-volume fly ash concrete blended with slag subjected to elevated temperatures.", Journal of Cleaner Production, v. 93, pp. 47-55, Abril 2015.

[42] JACKIEWICZ-REK, W., DRZYMALA, T., KUS, A., et al., "Durability of HPC subject to fire temperature impact", Archives of Civil Engineering, v. 62, n.4, pp 73-94, Jan. 2016.

[43] HUO, J., JUN, B., YU, Q., et al., "Effect of Microstructure Variation on damage evolution of concrete at high temperatures", ACI Materials Journal, v.113, pp. 547-558, Jan. 2016. 
[44] DRZYMALA, T., JACKIEWICZ-REK, W., TOMASZEWSKI, M., et al., "Effects of high temperature on the properties of high-performance concrete (HPC)", Procedia Engineering. v.172, pp. 256-263, 2017.

[45] SOUZA, A. A. A., MORENO JR, A. L. "Efeito de altas temperaturas na resistência à compressão, resistência à tração e modulo de deformação do concreto", Revista Ibracon de Estruturas e Materiais, v. 3, n. 4, pp. 432-448, 2010.

[46] CASTRO, A. L., TIBA, P. R. T., PANDOLFELLI, V.C. "Polypropylene fibers and their influence on the behavior of concretes exposed to high temperatures", Review Cerâmica v. 57, p. 22-31, 2011.

ORCID

Alisson Rodrigues de Oliveira Dias Felipe Alves Amancio

Maria Fabíola de Carvalho Rafael

Antônio Eduardo Bezerra Cabral https://orcid.org/0000-0002-9892-6834

https://orcid.org/0000-0002-1349-683X

https://orcid.org/0000-0001-8593-802X

https://orcid.org/0000-0001-6394-1164 